\title{
LA RELACIÓN \\ UNIVERSIDAD-EMPRESA EN BIZKAIA: UN ENFOQUE CUALITATIVO Y PROPUESTA DE MEJORA
}

\section{RESUMEN}

RESUMEN

La Fundación Ikertia, en colaboración con el Instituto de Economía Aplicada a la Empresa de la Universidad del País Vasco y con el apoyo de la Diputación Foral de Bizkaia, está realizando un estudio sobre los factores relevantes en las relaciones de investigación e innovación entre la universidad y la empresa en Bizkaia.

En este artículo se presenta un avance de los resultados, del estudio en el que se plantean algunos de los problemas y limitaciones en la relación de la universidad y Bizkaia.

\section{Palabras Clave}

Canales de relación Investigadores

Transferencia de conocimiento
Rosa Martínez*. Fundación IKERTIA**

$\mathbf{E}$ Fundación Ikertia está llevando a cabo un proyecto cuyo objetivo es el diagnóstico de las relaciones universidad - empresa en Bizkaia y la propuesta de nuevas metodologías de colaboración que mejoren dichas relaciones.

La creciente importancia de las actividades de I+D+i como factor estratégico y de adaptación a la nueva situación mundial ha hecho que se vuelvan los ojos hacia el modelo tradicional de generación de conocimiento e investigación: la universidad. El Science and Technology Policy Research de la Universidad de Sussex habla de la "tercera misión" de la universidad en la que incluye "la generación, uso, aplicación y explotación del conocimiento $u$ de otras capacidades fuera del entorno académico".

Es en este contexto en el que hemos de situar las relaciones Universidad - Empresa, como uno de los aspectos claves de cualquier economía regional competitiva. Si bien los beneficios mutuos de dicha relación son continuamente recordados y asumidos por todos los agentes, diversos factores siguen dificultando el entendimiento y la colaboración fluida entre el mundo académico y el mundo industrial.

\footnotetext{
* Colaboradora del Cluster del Conocimiento.

** Fundación dedicada a la agestión de I+d+i, a la certificación de proyectos $\mathrm{I}+\mathrm{d}+\mathrm{i}$ y a la formación de alto nivel.
} 
Muchos son los estudios, informes y estadísticas que a nivel europeo, estatal o regional intentan cuantificar las relaciones Universidad - Empresa: Estadística de I+D del INE, Informe COTEC de Tecnología e Innovación en España, Memoria de Actividades de I+D+i del Ministerio de Educación o a nivel regional, etc. Sin embargo, queremos destacar dos informes que por su especialización en el tema son referente en un estudio de este tipo: El informe de la Red OTRI Universidades, en el que todas las universidades españolas publican sus cifras de I+D y el Informe CyD 2006, en el que además de una compilación de los indicadores más relevantes de todos los estudios publicados, se añaden experiencias, opiniones y teorías muy interesantes para el enfoque de las relaciones universidad - empresa.

A pesar de la abundancia de datos cuantitativos y reflexiones sobre los indicadores más relevantes existentes, hemos de destacar la ausencia de datos concretos y homogéneos no sólo referentes a Bizkaia, sino a la Comunidad Autónoma Vasca. Las tres universidades vascas (UPV, Deusto y Mondragón) publican sus datos relativos al I+D y a la colaboración con el mundo empresarial de manera individual y generalmente de acuerdo a los indicadores establecidos por la Red OTRI de universidades. Por lo tanto, la primera conclusión extraída del análisis de las fuentes documentales secundarias es la necesidad de realizar un estudio más amplio y unificado de las relaciones Universidad Empresa en la CAV desglosado por territorio histórico, que sirviera de base para el establecimiento de una política regional de promoción de las relaciones Universidad - Empresa.

Sin embargo, nuestro estudio ha querido dar un enfoque cualitativo a la investigación, mediante entrevistas semi-estructuradas. Gracias a esta metodología, hemos podido obtener información muy valiosa directamente de investigadores, directores de unidades de I+D empresariales y directivos de empresas

\section{Abstract}

The Ikertia Foundation, together with The Applied Economics Institute in The País Vasco University, with the support of the Bizkaia Diputy Forum, which is studying the most relevant factors present in research and innovation relations between the University and the Company in Byzkaia. This report shows just the first steps and results of this study. Additionally, some problems and limitations in relationship between the University and Biskaia are presented.

\section{KEY WORDS}

Relationship canals Researchers Knowledge transfer. 
tecnológicas, directores de centros tecnológicos, responsables universitarios de relaciones con la empresa, cargos políticos, etc. que han evaluado la situación en Bizkaia, analizado problemas y hecho propuestas de mejora.

Los resultados han dado una larga lista de problemas y limitaciones que los entrevistados han señalado de forma reiterada. Factores como la falta de espíritu emprendedor en la universidad, la ausencia de difusión de las actividades que la propia universidad puede ofrecer a la empresa, las patentes, la diferencia de lenguaje, el gap generacional o la falta de tiempo, son algunos de los nuestro estudio ha recogido.Sin embargo queremos destacar dos de ellos, precisamente por su ausencia en los estudios cuantitativos y la falta de indi-cadores al respecto: los incentivos ofrecidos al personal universitario por trabajar con la empresa y los canales de relación.

En el primer caso, el actual sistema de evaluación de la actividad investigadora del profesor de universidad (los sexenios) está basado principalmente en criterios de calidad que normalmente son incompatibles con la investigación aplicada orientada a la transferencia de tecnología a la empresa (i.e. el número de publicaciones y la importancia de las revistas en las que aparezcan). Por ello, los profesores universitarios en particular en sus primeros años como investigadores, están más interesados en la investigación básica que en la aplicada por los beneficios económicos, de estabilidad $y$ de prestigio que les reporta dentro de la propia universidad.

En segundo lugar, el hecho de que todos los entrevistados señalen como principal forma de establecer un contacto Universidad - Empresa las relaciones personales, deja a la Universidad en una posición de debilidad, ya que, por una parte no se percibe a la universidad como agente innovador a tener en cuenta (la referencia es la persona no la institución) y por otro lado las empresas que no tengan esa relación personal quedan excluidas. Esto puede ser síntoma o bien, tal y como ha señalado alguno de los entrevistados, de la percepción de inaccesibilidad que se tiene de la Universidad (¿a quién dirigirse cuando se la quiere contratar?) o bien, de la escasa publicidad que la Universidad hace de sí misma y de sus capacidades (¿qué puede hacer la Universidad?).

Como complemento a esta primera parte de diagnóstico de la situación, estamos desarrollando actualmente una serie de propuestas operativas, en parte sugeridas por los propios interesados, en parte tomadas de modelos exitosos en la estructuración de relaciones Universidad - Empresa.

Las propuestas de actuación intentan abarcar todos los ámbitos que influyen en dicha relación: los canales de relación, los servicios profesionales, generación de enlaces estructurales, los perfiles de los equipos de investigación, los incentivos, la regulación normativa, las patentes y el ámbito institucional. Un vistazo a todos estos campos de actuación nos da una idea de la complejidad que encierran las relaciones Universidad - Empresa, y nos sugieren que la mejora de uno sólo de estos elementos no será suficiente. Así, aunque las instituciones elaboren políticas de apoyo y promoción, éstas no tendrán éxito si el perfil de los investigadores no es el adecuado, los profesores universitarios no están motivados o los canales de relación no se simplifican y se dan a conocer.

Por lo tanto, no hay un único responsable en la mejora de la transferencia de la investigación, el conocimiento y la innovación desde la Universidad a la Empresa, sino que todos los actores implicados deben asumir el reto que les toca. El modelo de la triple hélice: Universidad - Estado - Empresa y las organizaciones híbridas, está más al día que nunca. 


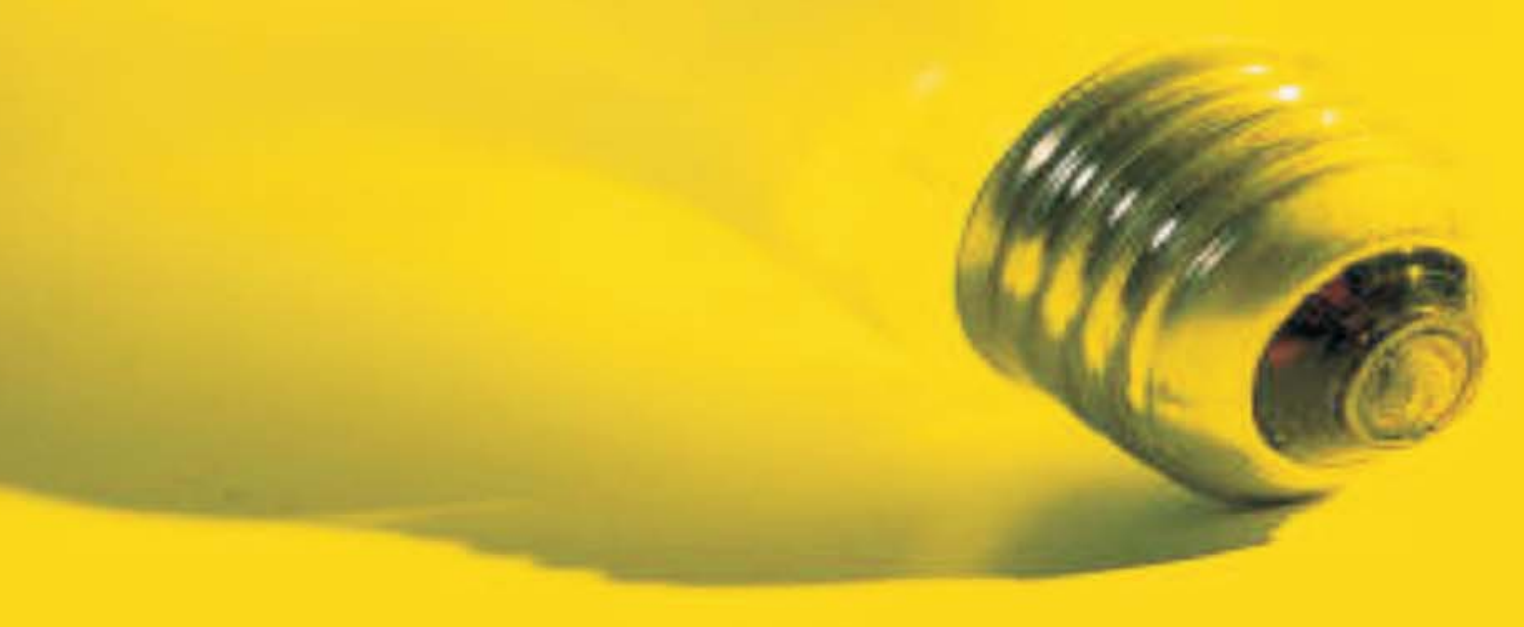

